\title{
POLUIÇÃO DO AR POR MATERIAL PARTICULADO EM ÁREA INTRAURBANA NO RIO DE JANEIRO: ASPECTOS METODOLÓGICOS
}

\section{Air pollution by particulate matter in intra-urban area in Rio de Janeiro: methodological aspects}

\author{
José Luiz Gatto Pereira ${ }^{1}$, Júlio Domingos Nunes Fortes ${ }^{2}$, Eduardo Monteiro Martins ${ }^{3}$
}

Recebido em 05 de janeiro de 2015; recebido para revisão em 09 de julho de 2015; aceito em 15 de setembro de 2015; disponível on-line em 11 de novembro de 2015.

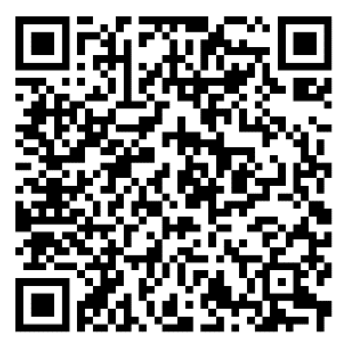

PALAVRAS CHAVE:

Material particulado;

Qualidade do ar;

Engenharia ambiental;

Saúde pública;

KEYWORDS:

Particulate matter;

Air quality;

Environmental

engineering;

Public health

RESUMO: O objetivo geral deste trabalho foi mensurar simultaneamente as concentrações de material particulado de $10 \mu \mathrm{m}, \mathrm{MP}_{10}$, em dois pontos de amostragem separados por $280 \mathrm{~m}$ dentro do campus de um Instituto de Pesquisa em Saúde, na cidade do Rio de Janeiro. Os dois pontos foram escolhidos com diferentes parâmetros físicos ambientais, como topografia, presença de bosque, proximidade de rodovia e construções. O objetivo específico foi detectar se há variações significativas nos valores das concentrações dos pares de medidas nessa baixa resolução espacial. Foram realizadas 16 amostragens durante dois meses, com duração entre $4 \mathrm{~h}$ e $6 \mathrm{~h}$ para cada exposição, em horário diurno de grande movimentação de veículos. Concluiu-se que a média dos valores das concentrações em cada ponto de amostragem apresentaram diferenças mesmo nessa pequena escala. Além disso, os valores estavam acima da média recomendada pela Organização Mundial da Saúde e considerados inadequados pelo programa de monitoramento do ar realizado na cidade do Rio de Janeiro.

ABSTRACT: The aim of this study was to measure simultaneously the particulate matter concentrations of $10 \mu \mathrm{m}, \mathrm{MP}_{10}$, in two sampling points separated by $280 \mathrm{~m}$ within the campus of an Institute of Health Research, in city of Rio de Janeiro. The two points were chosen with different environmental physical parameters such as topography, forest attendance, highway proximity and buildings. The specific objective was to detect if there are significant variations in the values of pairs of concentrations measured at the low spatial resolution. Were taken 16 samples for two months, lasting between 4 and 6 hours for each daytime exposure, at the time of large circulation of vehicles. It was concluded that the average values of the concentrations at each sampling point differences in this small scale. In addition, the obtained values were above average recommended by the World Health Organization and considered inadequate by program of monitoring in the air of government of Rio de Janeiro.

\footnotetext{
* Contato com os autores:

${ }^{1}$ e-mail: pereira.jlg@gmail.com ( J. L. G. Pereira )

Mestre em Engenharia Ambiental pela Universidade do Estado do Rio de Janeiro, UERJ. Professor da Faculdade de Engenharia da Universidade Iguaçu, UNIG, Nova Iguaçu, RJ, Brasil.

2e-mail : jfortes@uerj.br ( J. D. N. Fortes )

Doutor em Saúde Pública pela Escola Nacional de Saúde Pública Sérgio Arouca, Ensp, Fiocruz, RJ. Professor do Departamento de Engenharia Sanitária e Ambiental da Universidade do Estado do Rio de Janeiro, UERJ, RJ, Brasil.

3e-mail: edmmartins@gmail.com ( E. M. Martins )

Doutor em Físico-Química pela Universidade Federal do Rio de Janeiro, UFRJ, Brasil. Professor do Departamento de Engenharia Sanitária e Ambiental da Universidade do Estado do Rio de Janeiro, UERJ, RJ, Brasil.
} 


\section{INTRODUÇÃO}

A qualidade do ar nos centros urbanos pode variar significativamente de modo análogo à variação da temperatura no fenômeno chamado ilha urbana de calor. Esse fenômeno é uma anomalia térmica cuja temperatura de superfície do ar urbano varia em pequena escala geográfica por causa de parâmetros físicos ambientais, como excesso de área concretada, muitos trechos de pavimento asfáltico, material de construção que absorve calor, falta de áreas verdes, dificuldade de ventilação por causa do grande número de prédios altos e próximos uns dos outros que formam cânions. Estudos experimentais observaram e mensuraram valores diferentes da temperatura em pontos de amostragem próximos uns dos outros em um mesmo quarteirão dentro de bairros em grandes metrópoles (BATTERMAN, 2014; ERENO, 2010; COLTRI, 2007; GARTLAND, 2010; MORAES, 2005; SALDIVA, 2010; TRIGUEIRO, 2007). Nestes estudos a dimensão geográfica em questão é caracterizada como de baixa resolução, também chamada de ambiente intraurbano. Sua escala é da ordem de grandeza de algumas centenas de metros. Essa métrica é compatível com as proporções de pátios de grandes hospitais, campus universitários, parque industrial, áreas externas de grandes empresas, canteiros de obras, interior de pequenas comunidades, dentre outras com semelhantes proporções.

$\mathrm{Na}$ cinética das reações químicas da atmosfera, as substâncias que formam uma mistura gasosa têm as velocidades das suas reações influenciadas por diversos parâmetros físicos ambientais, como concentração das substâncias, temperatura do ar do entorno, natureza dos reagentes e tipos das superfícies de contatos com essa massa de ar (LENZI e FAVERO, 2009). Como as concentrações de diversos poluentes do ar fazem parte dessa composição na baixa atmosfera, dentro de um ambiente intraurbano, parâmetros como as topografias complexas (Isnard, 2004), os cânions urbanos (Solazzo et al, 2009; Taseiko et al, 2009), as áreas cobertas por asfalto e concreto (Coltri, 2007; Moraes, 2005), as colinas e qualquer outra rugosidade natural do solo (Borges, 2003) também impactam na qualidade do ar na baixa atmosfera.

Outros impactos são somados devidos a presença de pólo industrial (Martins, 2006; Pires, 2005) e a proximidade de rodovias com intenso fluxo de veículos (Batterman, 2014; Pattinson, 2014; Loureiro, 2005; Theurer, 1999). As sinergias entre eles provocam "mudanças climáticas, não necessariamente a provocada pelo aquecimento global" (Ereno, 2010), mas a que age na construção de um microclima no local - em pequenas dimensões geográficas (Ricklefs, 2003). Tais fenômenos se relacionam com as variações na intensidade e na direção dos ventos, com a incidência da radiação solar (Artaxo et al, 2005; Castanho, 1999) e demais questões sinóticas atmosféricas.

Nos últimos anos, as complexas interações que ocorrem entre os diversos parâmetros físicos ambientais e os processos de dispersão, deposição e ressuspensão de poluentes atmosféricos estão progressivamente sendo estudados pela abordagem da qualidade do ar em pequena escala geográfica. Além disso, essa forma de abordagem também contribui com estudos epidemiológicos (Batterman, 2014; Opas, 2008; Wilson et al, 2005; Wilson e Zawar-Reza, 2005), no que diz respeito aos impactos da poluição do ar na saúde de pequenos grupos de pessoas em locais específicos, cujos dados coletados in loco apresentam maior precisão em comparação com os das redes de monitoramentos regionais (HOEK et al, 2008; MADSEN, 2007; MENSINK et al, 2008; WEELER, 2008; SMARGIASSI et al, 2005).

O material particulado é um desses poluentes. Ele também pode viajar por longa distância (Prospero et al, 2004). Por exemplo, no ar urbano do Rio de Janeiro, pode ser oriundo das queimadas da biomassa da Floresta Amazônica (Artaxo et al, 2005; Freitas, 2005), ou das plumas de chaminés dos complexos industriais (Pires, 2005), ou dos ventos que trazem poluentes de países distantes - como a poeira que viaja pelo mar do Saara ao Caribe (PESTANO, 2006; PROSPERO et al, 2004). 


\subsection{JUSTIFICATIVA}

O estudo da qualidade do ar nessa baixa resolução espacial pode contribuir para definir estratégias de engenharia ambiental para adequações, adaptações e mitigações em infraestruturas construídas frente à poluição do ar localizada, e, também, para apoiar estudos epidemiológicos voltados para a saúde das pessoas que frequentam regularmente esses ambientes, ou residem nas proximidades.

\subsection{OBJETIVO}

Este trabalho, em linhas gerais, objetivouse em mensurar as concentrações de material particulado de $10 \mu \mathrm{m}, \mathrm{MP}_{10}$, em dois pontos próximos, dentro do campus de um Instituto de Pesquisa em Saúde, na cidade do Rio de Janeiro. Esta finalidade teve uma particularidade metodológica que foi mensurar esse poluente simultaneamente em dois pontos de amostragem afastados por $280 \mathrm{~m}$, e cada um deles com diferentes parâmetros físicos ambientais, como topografia, presença de bosque, proximidade de rodovia e construções. O objetivo específico foi detectar se há variações significativas nos valores das concentrações dos pares medidos nessa baixa resolução espacial.

\section{POLUIÇÃO INTRAURBANA}

No Brasil existem vários trabalhos de controle e monitoração dos impactos ambientais do material particulado, MP, por exemplo, seu impacto na saúde da população que mora em regiões metropolitanas (VORMITTAG et. al., 2014 A; VORMITTAG et. al., 2014 B; MONITORAR-RIO, 2012). O MP fica suspenso no ar e causa impacto negativo na saúde das pessoas, dos animais, desgastam e corroem as infraestruturas construídas, destroem vegetações, dentre outros danos.

Comprovadamente, a poluição do ar é uma complexa mistura de gases e MP com efeito carcinogênico, ao lado de perigosos poluentes conhecidos como asbesto, tabaco e radiação ultravioleta. Essa confirmação veio depois da consulta ao painel de especialistas organizado pela IARC, a agência do câncer da Organização Mundial da Saúde, OMS, com base em Lyon, na França (Cheng, 2013; WHO, 2013), mas ainda são poucos os estudos voltados para os impactos em ambientes intraurbano, com investigação das variações nos valores das concentrações do MP, e suas relações com grupos de pessoas que frequentam uma planta industrial ou partes internas de um bairro, ou na corrosão dos materiais utilizados em uma infraestrutura construída.

Estudos com relação à poluição do ar em ambientes externos localizados em meio intraurbano estão progressivamente ganhando espaço em periódicos que tratam do tema, bem como na mídia televisiva e escrita, inclusive, nos circuitos acadêmicos, com pesquisas voltadas para coletar dados através de instrumentos amostradores de volume de ar que podem ser transportados facilmente de um lugar a outro (MADSEN, 2007; MARTINS, 2006; LOUREIRO, 2005; PIRES, 2005; PATTINSON, 2014).

\subsection{MATERIAL PARTICULADO}

Grosso modo, entende-se como material particulado, MP, um conjunto de particulados, ou aerossóis, sólido ou líquido, suspenso no ar. Em geral é invisível a olho nu pois possui dimensão entre $0,001 \mu \mathrm{m}$ e $100 \mu \mathrm{m}$. Esses particulados são grandes comparados com o tamanho das moléculas dos gases traço, ou de outros gases presente na atmosfera, pois as moléculas desses gases possuem valores típicos de 0,0001 $\mu \mathrm{m}$ a 0,001 $\mu \mathrm{m}$ (BAIRD, 2011; SEINFELD e PANDIS, 2006). Embora alguns particulados apresentem forma diferente da esférica, é convencional tratar a totalidade deles como se apresentassem essa forma. Esse diâmetro aerodinâmico é uma propriedade relevante em seus estudos. Qualitativamente, os particulados são classificados dependendo de o seu diâmetro ser maior ou menor que $2,5 \mu \mathrm{m}$. Atualmente, o MP com diâmetro entre 0,001 $\mu \mathrm{m}$ e 0,1 $\mu \mathrm{m}$ é chamado de partícula ultrafina. Essas dimensões caracterizam o comportamento dos aerossóis suspensos na atmosfera. Por exemplo, quando gotículas de água 
presentes na atmosfera se aglutinam e formam particulados maiores do que $100 \mu \mathrm{m}$ precipitam em direção ao solo rapidamente (hidrometeoro). Outro exemplo da utilização dessa classificação é com relação à saúde pública, em que particulados abaixo de $10 \mu \mathrm{m}$ podem atingir os alvéolos pulmonares e carregar com eles outros poluentes perigosos. Os particulados, dependendo das suas fontes, podem conter em suas composições substâncias à base de enxofre e oxigênio, que em contato com a água da atmosfera produz, ao atingir mais de $100 \mu \mathrm{m}$ de tamanho, chuvas ácidas que corroem infraestruturas construídas e prejudicam as espécies vivas (Baird, 2011; Seinfeld e Pandis, 2006), inclusive a alcalinidade do concreto e suas armações de vergalhões (AGOPYAN e JOHN, 2011; GJORV,2015).

Dentre algumas fontes naturais desses aerossóis estão os sais marinhos, as poeiras trazidas por ventos, os pólens, as emitidas por vulcões, ou originadas nas biodegradações dos vegetais e dos animais, e nos processos das evapotranspirações das florestas (PESTANO, 2006; PROSPERO et al, 2004).

Entre as fontes antropogênicas, os particulados são produzidos nas queimadas de biomassa; nas emissões de motores veiculares à base de combustíveis fósseis; na produção, manejo, disposição e incineração de resíduos sólidos rurais e urbanos; nas emissões originadas no processamento energético em parques industriais, como incineradores, chaminés; na ressuspensão de particulados do solo por motivo de tráfego nas vias públicas; nos gastos dos pneus; nas atividades da construção civil etc.. Muitos outros processos antropogênico também são fontes de aerossóis, por exemplo, respiração humana e de animais, tosses, espirros, o ato da fala, a limpeza de ambientes ou de objetos por jateamento seja de água ou areia.

Efetivamente, o MP é formado por sulfatos, nitratos, amônia, material orgânico, espécies de cristais, sais marinhos, metais óxidos, íons de hidrogênio e água (BAIRD, 2011; SEINFELD e PANDIS, 2006). Espécies como sulfatos, amônias, orgânicos (carbono elementar), e certos metais de transição são encontrados nas partículas mais finas $\left(\mathrm{MP}_{2,5}\right)$. Materiais cristalizados, incluindo, silício, cálcio, magnésio, alumínio, ferros, partículas orgânicas biogênicas (pólen, esporos, fungos, bactérias, vírus, fragmentos de plantas) são usualmente encontrados nas partículas mais grossas $\left(\mathrm{MP}_{10}\right)$ Figura 1.

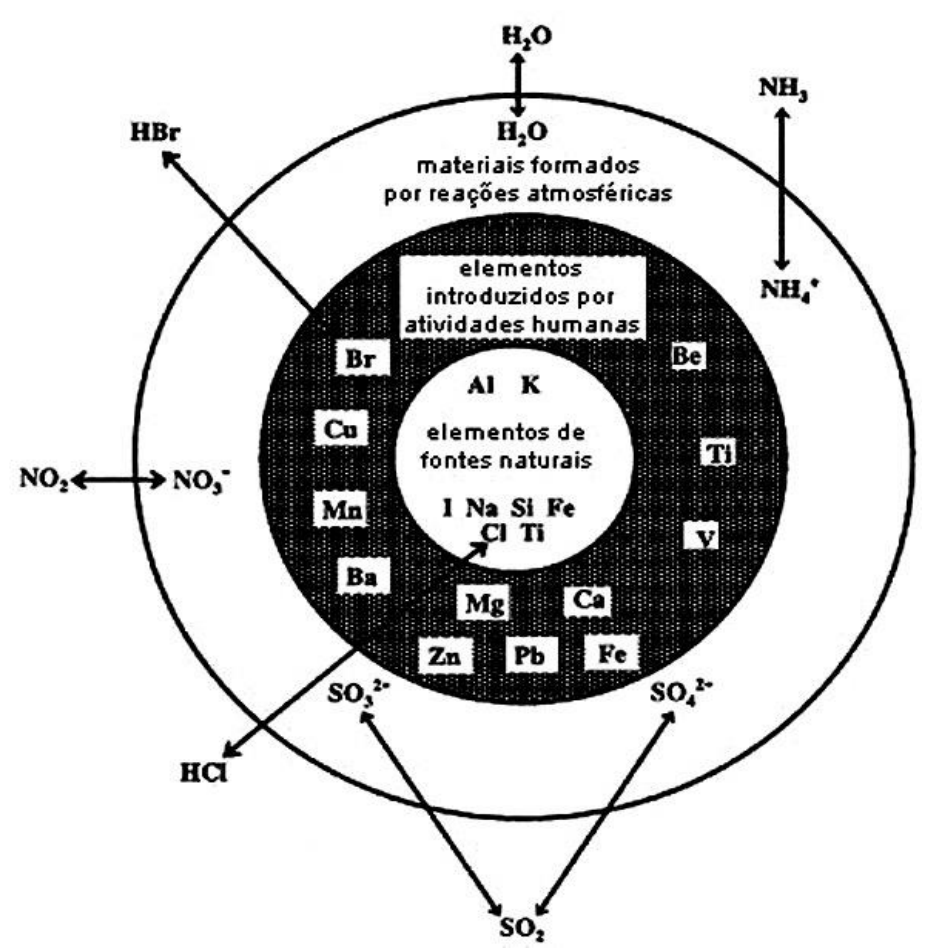

FIGURA 1: Esquema exemplo de aerossóis inorgânicos com um cinturão de vapor de água. FONTE: MANAHAN, 2001, modificada. 
O efeito de parâmetros meteorológicos (temperatura, umidade relativa, radiação solar, pressão, velocidade do vento, direção do vento e precipitação), e, suas relações com ambientes externo e interno, são, também, discutidos na temática da qualidade do ar intraurbano, através dos seus impactos nas concentrações do MP. As ocorrências de estagnação, de recirculação e ventilação natural são os efeitos que mais impactam nos valores das concentrações desse poluente na baixa atmosfera. As concentrações máximas de MP são observadas nas condições atmosféricas estagnadas (CHITHRA e SHIVA NAGENDRA, 2014).

De certo modo, mesmo em MP com dimensões em torno de $10 \mu \mathrm{m}$ coletados suspensos no ar há percentuais importantes das partículas finas e ultrafinas (BAIRD, 2011, MANAHAN, 2001; SEINFELD e PANDIS, 2006). Além disso, esses particulados nunca estão sozinhos pois podem carrear muitos microrganismos em sua composição, os chamados bioaerossóis.

\subsection{ASPECTO SOCIOECONÔMICO E MORBI- MORTALIDADE}

Atualmente, no Brasil, a poluição do ar por MP mata mais do que a Aids e o trânsito juntos, apenas na cidade de São Paulo. Nesta cidade brasileira, as doenças provocadas pela poluição do ar causam cerca de 9 mortes por dia e custo anual em torno de US\$ 1,5 bilhão para tratar as doenças provocadas por essa poluição (PARAJARA, 2008; RAMOS, 2008).

No Rio de Janeiro, a pesquisa feita pelo Instituto Saúde e Sustentabilidade, que foi realizada entre 2006 e 2012, mostrou que o índice de poluição do Rio é duas vezes maior do que o recomendado pela OMS. O índice é quase igual à média de poluição no Estado de São Paulo apresentada no mesmo período. A pesquisa apontou que há "36.194 mortes e 65.102 internações na rede pública de saúde devido à poluição, sendo 14 mortes por dia em todo estado, representando um gasto público de $\mathrm{R} \$ 82$ milhões, entre 2006 e 2012" (VORMITTAG et. al., 2014 A). Os municípios mais castigados são: Duque de Caxias,
Itaboraí, Nova Iguaçu, Macuco, Resende e Porto Real. Já aqueles com maior risco de morte são: Macuco, Duque de Caxias, Nova Iguaçu, Itaboraí, Barra Mansa e a capital, por "apresentarem maiores níveis de poluição, maior número de dias poluídos no ano e maiores taxas de mortalidade" (Vormittag et. al., 2014 A). Em 2011, em comparações com outras mortalidades, o número de mortes atribuídas à poluição foi cerca de "uma vez e meia maior que os 3.044 óbitos por acidentes de trânsito, quase três vezes maior que as mortes por câncer de mama ou decorrentes da AIDS e quase sete vezes maior que falecimentos por câncer de próstata" (Vormittag et. al., 2014 A).

\subsection{ASPECTOS LEGAIS}

No município do Rio de Janeiro o exame periódico da qualidade do ar utiliza o Índice de Qualidade do Ar, IQA, para simplificar o processo de divulgação entre os diversos segmentos da população. O Programa MonitorAr-Rio (2012) optou por esse padrão porque já era utilizado pela Companhia de Tecnologia de Saneamento Ambiental do Estado de São Paulo, CETESB, desde a década de 1980, e que foi baseado em experiências do Canadá e EUA (MONITORAR-RIO, 2012). A estrutura de IQA adotada possibilita a comparação com os limites legais de concentrações para o $\mathrm{MP}_{10}$, e outros poluentes, que são monitorados pelas estações da rede municipal. A divulgação é feita por meio de boletins diários de cada estação. Os critérios para episódios agudos são estabelecidos pela Resolução CONAMA n. 003 de 28 de junho de 1990 (Figura 2).

Com relação ao controle da poluição por MP e seu nexo epidemiológico no Brasil, o Ministério da Saúde, em 1999, a partir do projeto de fortalecimento da Vigilância Epidemiológica, criou a Vigilância em Saúde Ambiental. Logo, em 2001, foi criada a Vigilância em Saúde Ambiental Relacionada à Qualidade do Ar - VigiAR (Brasil, 2007), no âmbito da Secretaria Nacional de Vigilância em Saúde, SVS/MS, do Sistema Único de Saúde, SUS. Esse programa, não só considerou o MP como um indicador de saúde ambiental, mas também o nexo com morbidade e mortalidade, 
registrado no Código Internacional de Doenças, CID10. Estes dados são registrados no banco de dados nacional, chamado TABWIN, do Departamento de Informática do SUS, DATASUS, e dá subsídios para a pactuação de limites na concentração desse indicador, que são os mesmos limites recomendados pela Organização Mundial da Saúde, OMS.

O programa VigiAr em seu "Relatório Final - Reunião de Avaliação das Metas 2006 e Pactuação de Ações para 2007" (Brasil, 2007) realizado em julho de 2007, em Brasília, apresentou a Tabela 1 traduzida diretamente do Sumário de Análise de Risco da OMS (Who, 2006), que foi atualizado em 2006, e ainda em vigor. O sumário traz em seu conteúdo recomendações dessa organização para a revisão dos padrões de qualidade do ar. Define Objetivos Intermediários (OI), que são valores máximos toleráveis das concentrações desse poluente que devem ser atingidos através de uma redução progressiva. A redução implica na diminuição dos riscos para efeitos agudos e crônicos desse poluente sobre a saúde das pessoas.
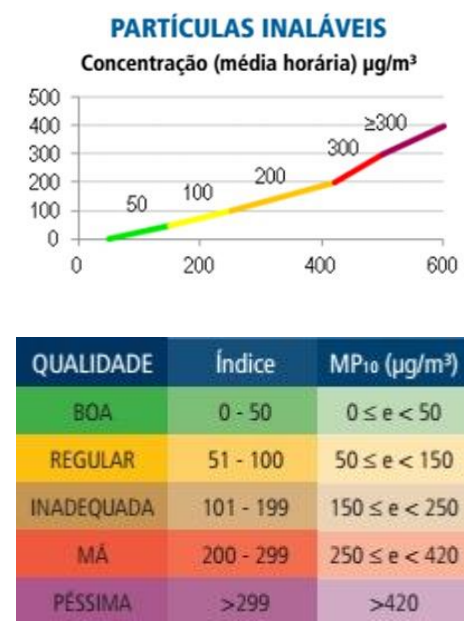

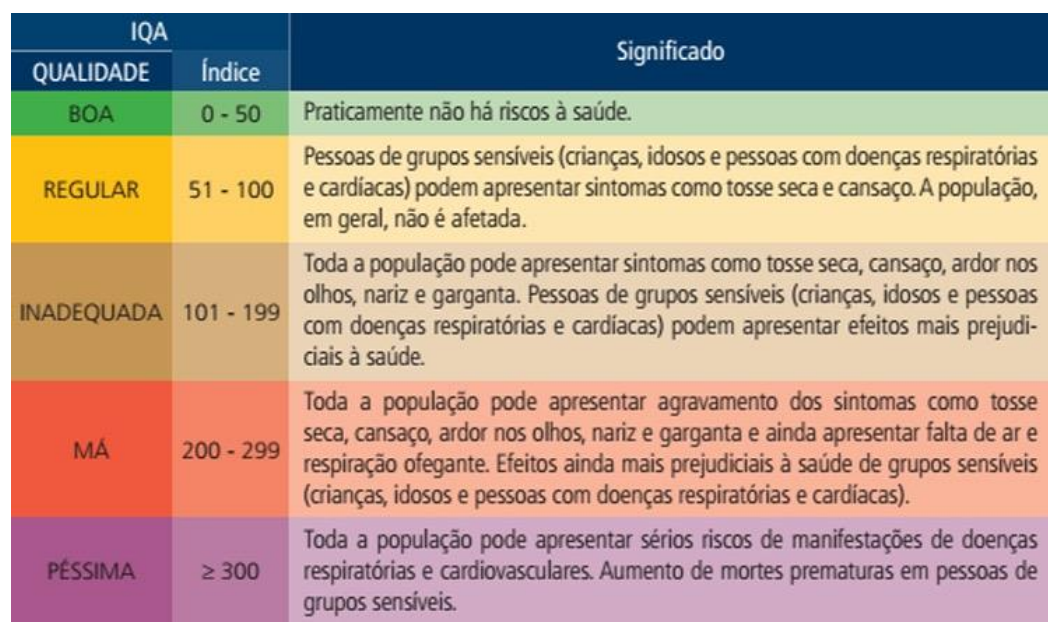

FIGURA 2: Critérios de classificação do Índice de Qualidade do $\operatorname{Ar}$ (IQA).

FONTE: Adaptado do Relatório MONITORAR-RIO, do Programa de Monitoramento do Município do Rio de Janeiro, 2012.

TABELA 1: Padrões de qualidade do ar e OI para material particulado: média diária em $\mu \mathrm{g} \cdot \mathrm{m}^{-3}$.
Nível da média diária
$\mathrm{MP}_{10}\left(\mu \mathrm{g} \mathrm{m}^{-3}\right)$
$M P_{2,5}\left(\mu \mathrm{g} \mathrm{m}^{-3}\right)$
Fundamentação

Objetivo Intermediário - 1 (OI - 1) da OMS

Objetivo Intermediário - 2

$$
(\mathrm{OI}-2) \text { da OMS }
$$

$$
\text { (OI - 3) da OMS }
$$

Guia de qualidade do ar da OMS (GQA)
50
Baseado em coeficientes de risco publicados em estudos multicêntricos e metanálise (incremento de cerca de $5 \%$ de mortalidade de curto prazo).

Baseado em coeficientes de risco publicados em estudos multicêntricos e metanálise (incremento de cerca de 2,5\% de mortalidade de curto prazo).

Incremento de cerca de $1,2 \%$ de mortalidade de curto prazo.

Baseado na relação entre os padrões diário e anual de material particulado.

FONTE: VIGIAR - Vigilância em Saúde Ambiental Relacionada à Qualidade do Ar. Ministério da Saúde. Secretaria de Vigilância em Saúde. (BRASIL, 2007). 


\section{METODOLOGIA}

O MP é emitido tanto por fontes fixas, como parque industrial, quanto por fontes móveis, como veículos com motores à combustão. Sua medida é representada por uma quantidade de particulado presente em um dado volume de ar que pode ser segregado por tamanho. Como a matéria envolvida no particulado não é homogênea, nenhuma massa molar pode ser atribuída. Portanto, suas concentrações são dadas em termos da sua massa, em vez do seu número de mols. A unidade usual é o micrograma de material particulado por metro cúbico de $\operatorname{ar}\left(\mu \mathrm{g} \mathrm{m}^{-3}\right)$ (BAIRD, 2011; SEINFELD e PANDIS, 2006).

\subsection{CARACTERIZAÇÃO DO LOCAL DE ESTUDO}

Resolveu-se mensurar os valores das concentrações do material particulado com tamanho aerodinâmico até $10 \mu \mathrm{m}$, chamado $\mathrm{MP}_{10}$, que são partículas inaláveis. O local escolhido para os dois pontos de amostragem foi o situado na Região Metropolitana do Rio de Janeiro, RMRJ, no bairro de Manguinhos, na área externa do Instituto Nacional de Infectologia, Ini, situado no campus da Fundação Oswaldo Cruz, Fiocruz/RJ. Esses pontos se localizaram ao longo da via expressa de maior concentração desse poluente na RMRJ, em um eixo rodoviário de aproximadamente $40 \mathrm{~km}$ que liga o Centro do RJ à Nova Iguaçu (Figura 3).

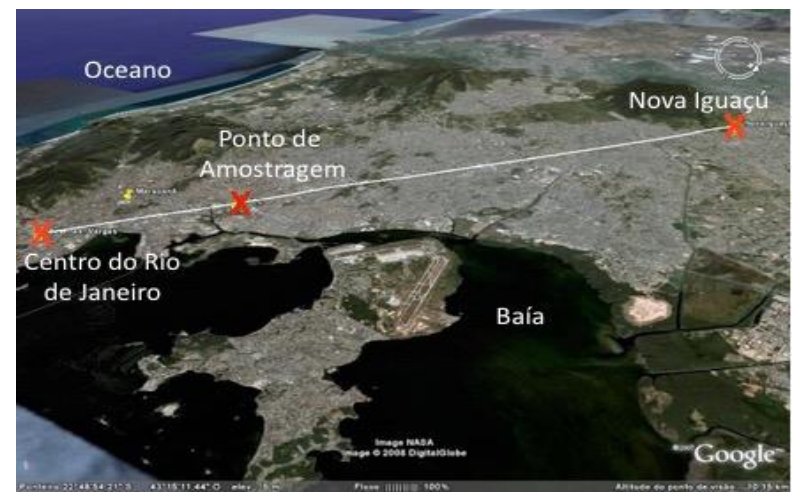

FIGURA 3: Via expressa escolhida. RMRJ. FONTE: Adaptado do software Google Earth (edição de uso livre).

Nessa via, os dois pontos próximos escolhidos ficaram dentro do campus da Fiocruz, que está mais próximo do Centro do RJ do que de
Nova Iguaçu. A Fiocruz está situada no bairro de Manguinhos, entre os bairros de Bonsucesso e Caju. Nessa veia urbana se encontra o trecho crítico da Avenida Brasil, que possui o maior fluxo de veículos da RMRJ. Ela apresenta um tráfego com o quantitativo de mais de 270.000 veículos por dia. Essa via contribui com mais de $23 \%$ da poluição do ar na RMRJ, apenas com o poluente $\mathrm{MP}_{10}$. As fontes móveis contribuem com $77 \%$ da poluição do ar (MONITORAR-RIO, 2012). Observa-se na Figura 3 que os maciços - canto superior esquerdo interceptam os ventos marítimos, o chamado vento sudoeste. Esse eixo é um dos principais corredores de poluentes, e conta apenas com as brisas matinal e noturna oriundas da Baía da Guanabara para dispersão dos poluentes suspensos no ar.

Os dois pontos escolhidos dentro da Fiocruz possuem parâmetros físicos ambientais diferenciados. Um ponto, o ponto A, estava livre de obstáculos de infraestruturas construídas, topografia plana, cobertura de grama, incidência direta da radiação solar, fora do bosque, mas ao lado da rodovia. O outro ponto, o ponto $B$, estava blindado por um pequeno bosque, próximo de infraestrutura construída e com concreto como cobertura do solo (Figura 4).

Ao determinar esses pontos, a demarcação entre eles baseou-se numa grade virtual horizontal (bidimensional) elaborada de forma que os incluíssem. Construída em forma de malha quadrada, cada unidade ou célula tinha tamanho fixo, em torno de $200 \mathrm{~m}$ de lado. A célula escolhida está representada pela linha diagonal que liga os pontos A e B (Figura 4). O ponto A está ao nível do mar, a $100 \mathrm{~m}$ da rodovia Avenida Brasil. 0 ponto B está situado em frente ao Instituto Nacional de Infectologia, na varanda aberta do seu prédio principal, aproximadamente $20 \mathrm{~m}$ acima do nível do ponto $A$. Os pontos distam aproximadamente $280 \mathrm{~m}$ (Figura 4).

O posicionamento dos pontos na diagonal da célula escolhida coincidiu com a direção dos ventos predominantes - do ponto $A$ no Leste até o ponto $B$ no Oeste. Esses ventos variam de sentido dependendo da hora do dia ou da estação 
do ano. Os movimentos verticais da massa de ar dependem da variação da temperatura e da pressão, com a altitude.

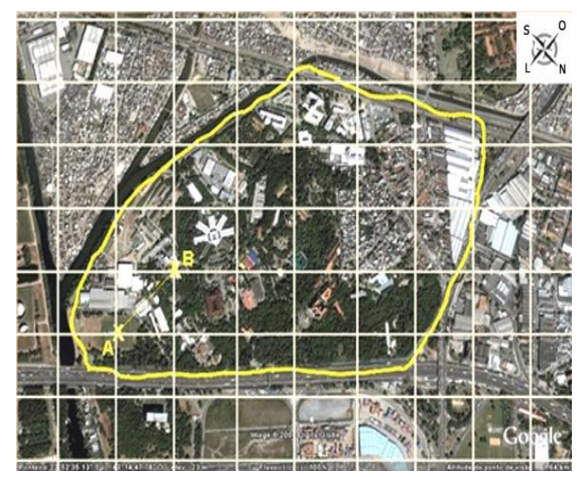

[a]

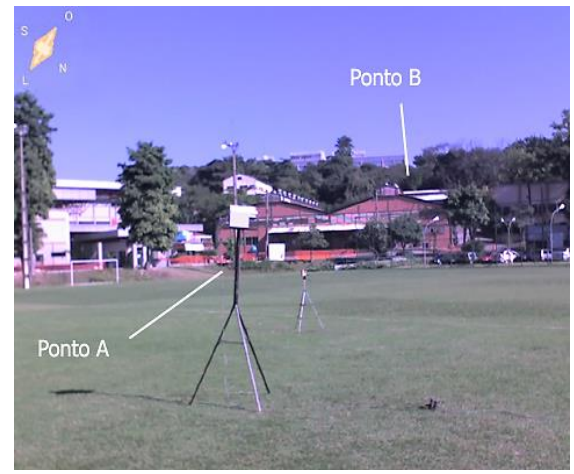

[b]

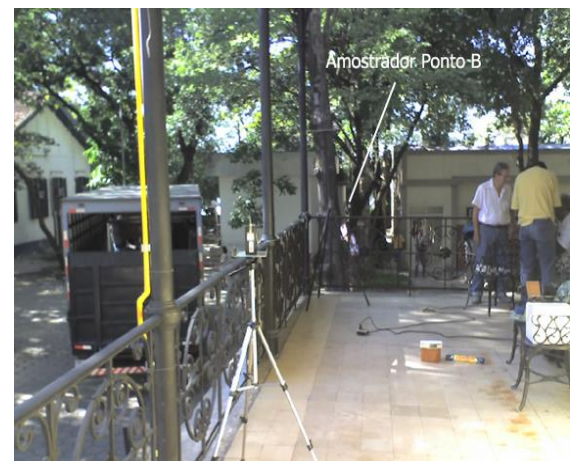

[c]

FIGURA 4: [a] Mapa ilustrativo da região estudada com a resolução da grade de células $2 \mathrm{~km} \times 1 \mathrm{~km}$. Na área circunscrita está o campus da Fiocruz/RJ; [b] o amostrador de ar no tripé em primeiro plano (ponto A) e o local do segundo ponto; [c] a varanda imersa no bosque (ponto B). FONTE: Figura 4 [a] adaptada do software Google Earth - edição uso livre.

\subsection{INSTRUMEMENTAÇÃo}

Os equipamentos utilizados na campanha de amostragem deste estudo (Figura 5), foram 2 (dois) Amostradores de Grande Volume - Marca SIBATA - modelo HVS 500 - S, que foram colocados um em cada ponto de amostragem e funcionaram simultaneamente; 1 (uma) Balança Analítica Mettler Instruments, modelo AX 205, sensibilidade de $0,01 \mathrm{mg}$, que foi utilizada para mensurar a massa dos filtros dos amostradores antes e depois de cada amostragem; 2 (dois) Tripés de 2,10 m de altura, que foram os suportes dos amostradores para mantê-los numa altura razoável para coleta do ar local; 2 (dois) Patamares de alumínio como bandejas para Tripé, que foram as bandejas utilizadas e aparafusadas no topo de cada tripé para assentar os amostradores; 1 (um) Mala c/ 2 Cabos de Força c/ Extensões de $60 \mathrm{~m}$ cada, esta mala foi utilizada para o transporte dos amostradores do laboratório até os pontos de amostragens, e, os cabos de forças e as extensões foram necessárias para conectar os amostradores ao ponto de energia elétrica mais próximo.

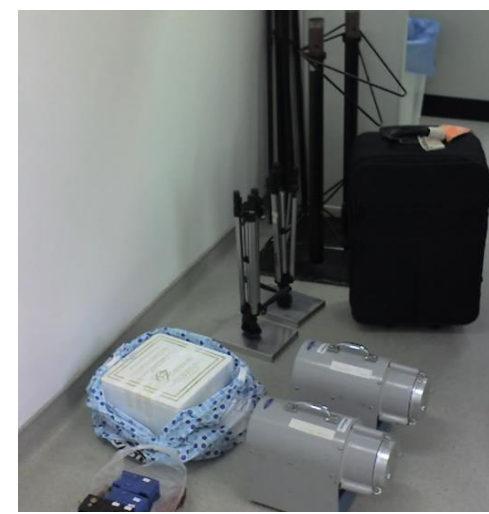

[a]

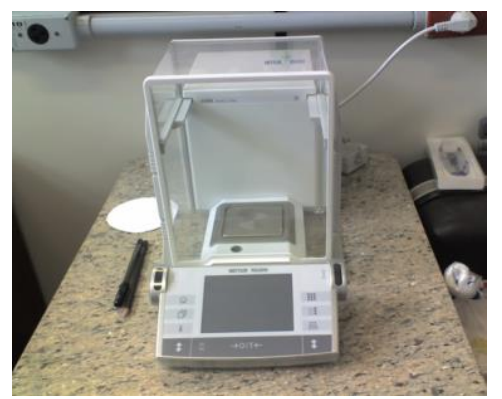

[b]

FIGURA 5: [a] Kit de campanha; [b] Balança de precisão com o filtro de fibra na mesa da balança.

Os insumos utilizados foram 2 (dois) Filtros de Fenda c/ corte de $10 \mu \mathrm{m}$, que foram utilizados para permitir que apenas o material particulado desta dimensão fosse coletado pelos amostradores; 20 (vinte) Filtros de Fibra de vidro de 110mm - E100 - SIBATA - Padrão Normal, que foram utilizados para reter o material particulado, 
para as pesagens antes e depois das amostragens, e avaliação do quantitativo; 2 (duas) caixas de Isopor com capacidade de $6 \mathrm{~L}$, que foram utilizadas para o transporte dos filtros de fibra em condições de isolamento do ar fora do local de amostragem; 3 (três) Potes de vidro não transparentes com capacidade de $1 \mathrm{~L}$ com tampa hermética, que foram os recipientes em que os filtros de fibra ficavam armazenados; 1 (um) Tubo 250 g de Silicone Gel, que foi passado nos filtros de fenda para melhor aderência e vedação para os filtros de fibra; 3 (três) rolos de papel alumínio, que foram utilizados no transporte e armazenamento dos filtros de fibra; 4 (quatro) rolos de fita teflon, que foram utilizadas nas roscas dos amostradores para maior isolamento dos anéis de vedação dos filtros de fenda.

\subsection{PROCEDIMENTOS METODOLÓGICOS DE MONITORAMENTO E AVALIAÇAO}

As coletas foram feitas utilizando dois amostradores de grandes volumes de ar que são bombas de sucção com vazão constante. Há um disco vazado com diâmetro de $110 \mathrm{~mm}$ na entrada de ar, acoplado com um filtro de fenda para corte de partículas de até $10 \mu \mathrm{m}$ (parte frontal). 0 ar passa por essa fenda até encontrar o filtro de fibra de vidro, onde o MP é retido (Figura 6).
Nas coletas com amostradores de grandes volumes os filtros de fibra de vidro devem garantir eficiência de $99 \%$, baixa reação com material corrosivo, baixa higroscopia, $\mathrm{pH}$ superior a 7,5 , resistência a temperaturas de até $540^{\circ} \mathrm{C}$, resistência a tensões (não se rompe facilmente com o manejo) e possibilidade de erros entre 0,3 e 3,0 $\mathrm{g} \mathrm{m} \mathrm{m}^{-3}$ (NBR 13412, ABNT 1995; NBR 9547, ABNT 1997). Essas normas recomendam metodologias para obtenção das concentrações de partículas inaláveis por meio de amostradores de grandes volumes acoplado a um separador inercial de partículas (filtro de fenda).

No processo de coleta da amostra de MP, primeiramente, o filtro é desumidificado, depois pesado em uma balança eletrônica de precisão $0,01 \mathrm{mg}$. O filtro é então colocado em uma caixa do tipo de CD Rom, embrulhado em papel alumínio e transportado em recipiente térmico (caixa de isopor) até o local da amostragem. Após esta etapa, cujo tempo escolhido foi em torno de $4 \mathrm{~h}$ a $6 \mathrm{~h}$ de exposição por dia, o filtro é novamente armazenado e embrulhado com papel alumínio, e guardado no dessecador até o transporte de volta para o laboratório, onde foi pesado finalmente (Figura 7), e a massa do material retido é obtida por diferença entre a pesagem inicial e final.

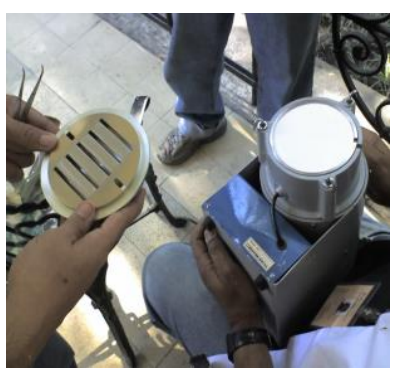

[a]

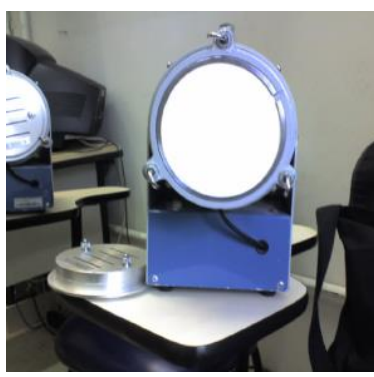

[b]

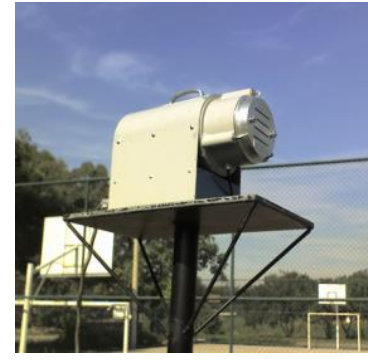

[c]

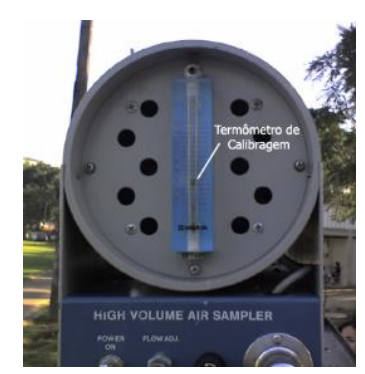

[d]

FIGURA 6: [a] Montagem da bomba (filtro de fenda na mão e filtro de fibra já na bomba); [b] bomba com o filtro de fibra colocado e filtro de fenda ao lado; [c] bomba montada no tripé; e [d] termo-rotâmetro: sensor de calibragem do volume do ar coletado (parte traseira do amostrador). 


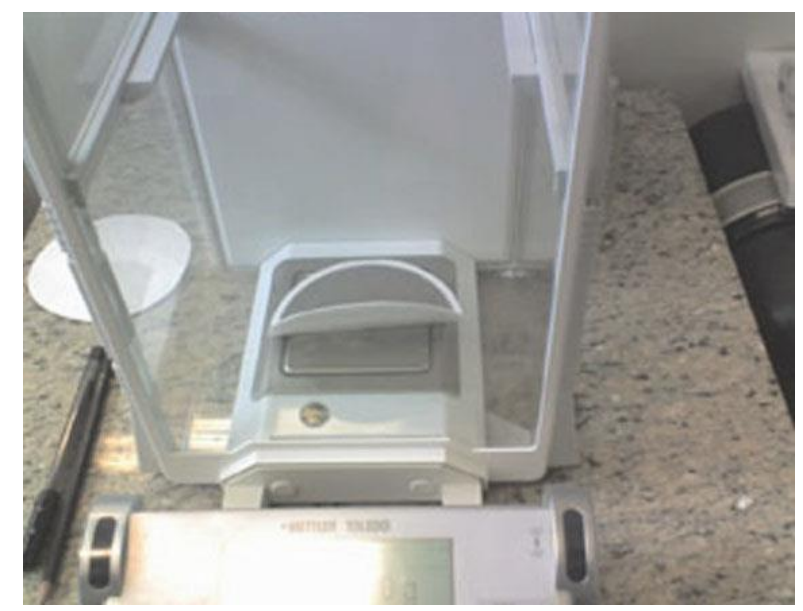

FIGURA 7: Filtro posicionado na balança com a marca circular em escuro provocado pelos particulados que ficaram retidos na fibra.

A vazão de operação utilizada nos dois amostradores, em ambos os pontos de amostragem, foi constante, de aproximadamente $500 \mathrm{~L} \mathrm{mim}^{-1}$, ou seja, $30 \mathrm{~m}^{3} \mathrm{~h}^{-1}$. Acima desse valor o amostrador apresentou alguma instabilidade. A vazão foi controlada permanentemente por meio de um termo rotâmetro localizado na parte de trás do amostrador, cujo visor é em forma de ampola de vidro graduada (Figura 6d). Após a parada do equipamento as telas de entrada de ar, o filtro de fenda e o equipamento eram higienizados. $O$ filtro de fenda antes da amostragem era untado com silicone gel para reter MP maiores que $10 \mu \mathrm{m}$. Nas medições, uma pessoa estava em cada ponto de amostragem para ligar e desligar a bomba de forma sincronizada por meio do uso de telefones celulares.

Antes das pesagens dos filtros de fibra de vidro era observado se a balança estava marcando zero. Os filtros eram colocados no prato da balança com pinças, sem o contato manual. Após colocar o filtro no prato aguardava-se a estabilização do marcador digital, e anotava-se o valor da massa nos formulários de campo, pois a pesagem inicial e as identificações dos filtros estavam ali anotadas, junto com os dados das condições atmosféricas do momento da amostragem (direção e velocidade do vento, temperatura, etc.).

A determinação da concentração do material particulado de até $10 \mu \mathrm{m}$ (Braga et al, 2005; Frondizzi, 2008; Vesilind e Morgan, 2011) foi feita por meio da razão entre a diferença de massa do filtro, e a vazão total, utilizando os valores já convertidos para $\mu \mathrm{g} \cdot \mathrm{m}^{-3}$, através da aplicação da Equação 1.

$$
C_{M P_{10}}=\frac{M_{f}+M_{i}}{V_{a}}
$$

Em que:

$\mathrm{C}_{\mathrm{MP10}}$ - Concentração de material particulado em

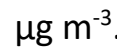

$M_{f}-$ Massa final em $\mu g$.

$M_{i}$ - Massa inicial em $\mu \mathrm{g}$.

$V_{a}$ - Volume de ar que foi coletado no período de cada amostragem em $\mathrm{m}^{3}$.

Durante os dois meses de campanha foram realizadas 16 amostragens, oito em cada ponto, sempre entre $10 \mathrm{~h}$ e $16 \mathrm{~h}$. As medições aconteceram apenas nos dias de céu claro, poucas nuvens, para não haver deposição no solo ou transporte anormal do poluente por motivo de chuva ou de ventos fortes.

Os amostradores eram ligados $\mathrm{e}$ desligados simultaneamente. Os manuseadores dos amostradores ficavam conectados por celular para sincronizar o início e o término da amostragem diurna. Ao término de cada medição os filtros de fibra eram retirados, armazenados e transportados ao laboratório para realizar a pesagem. O amostrador era higienizado e guardado para o outro dia de coleta.

\section{DISCUSSÃO DOS RESULTADOS}

Este estudo realizou 16 amostragens (oito em cada ponto analisado). A média aritmética obtida no Ponto $A$ (Campo) foi igual a $108,50 \mathrm{mg} \mathrm{m}^{-3} \mathrm{e}$, no Ponto B (Ini) foi igual a $138,20 \mathrm{\mu g} \mathrm{m}^{-3}$. Observou-se diferenças nos valores das concentrações entre os dois pontos de amostragem.

No gráfico da Figura 8 está a evolução simultânea das medições de MP nos dois pontos de amostragem. Observam-se picos e vales não coincidentes. A média aritmética dos valores das concentrações no ponto B - área inserida no bosque, próxima da construção, mais distante da 
Avenida Brasil etc. é maior que a média no Campo, ponto A. A primeira medição neste ponto registrou um salto abrupto no valor da concentração. Inclusive, aconteceu outro salto na 6a amostragem do ponto $A$.

Os valores das concentrações que foram encontrados se relacionam não apenas com eventos adversos que ocorreram em cada ponto, como também com os parâmetros físicos ambientais escolhidos. Por exemplo, foi observado que há diferenças nos valores das concentrações do poluente entre os dois pontos de amostragem tanto nos dias em que ocorreram estagnação dos ventos, céu limpo e fluxo normal de veículos quanto nos dias em que ocorreram eventos adversos diferentes nos locais de amostragem, como o dia em que um veículo com motor diesel estava parado realizando uma entrega no Ponto B com o motor ligado próximo ao amostrador. Tal fato foi detectado pela sensibilidade do equipamento em registrar um pico no valor da concentração.

$\mathrm{O}$ aspecto metodológico mais relevante nos resultados das medições das concentrações de material particulado em pequena escala geográfica, próximo ao solo e em ambiente intraurbano é que os amostradores de grandes volumes são sensíveis o suficiente para registrar eventos como atividade de limpeza com varrição, pequenas obras, carros automotores movidos por óleo diesel, ou gasolina, estacionados próximos e com motores ligados, pequenas obras em pavimento ou em infraestrutura predial, ação dos ventos, da chuva e demais intempéries do clima.

Este estudo, metodologicamente, não evitou todas as possíveis intervenções de eventos adversos que poderiam ocorrer nos pontos de amostragem, para que apenas os parâmetros físicos selecionados pudessem prevalecer. Os critérios para acionar os amostradores foram apenas o de céu limpo e sem previsão de chuvas para as $6 \mathrm{~h}$ que durariam os funcionamentos dos dois equipamentos nos dias de coleta. Os eventos que iam ocorrendo durante as medições foram registrados na planilha de campo para confrontar com as possíveis variações nas concentrações obtidas após a pesagem final dos filtros.

A simplicidade metodológica permitiu obter variações nos valores das concentrações em pequena escala. Além disso, se mostrou como este poluente não só é proveniente de muitas fontes, como também ele pode ser amplificado por eventos que ocorrem próximos das pessoas que frequentam pátios de empresas, escolas, hospitais, campus universitário e demais deslocamentos em pequenos trechos intraurbano.

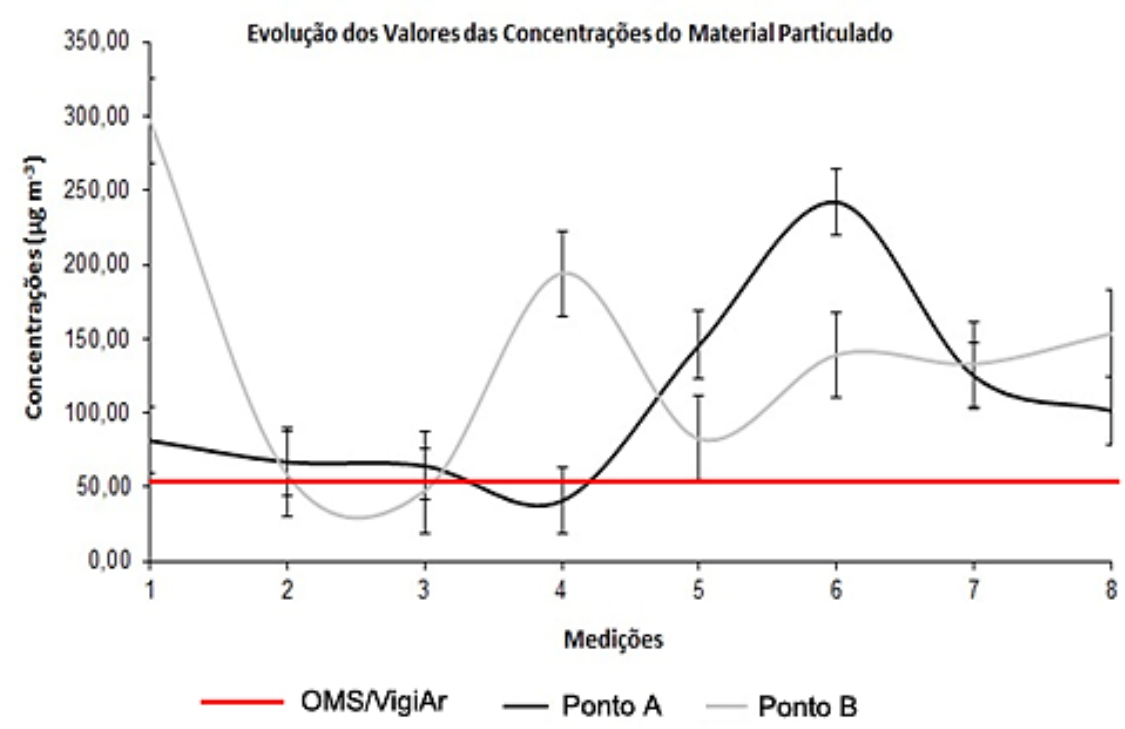

FIGURA 8: Resultado do monitoramento de $\mathrm{MP}_{10}$, com os valores das concentrações em $\mu \mathrm{g} \mathrm{m}^{-3}$. 


\section{CONCLUSÃO}

Um aspecto relevante foi que os valores médios das concentrações estavam acima dos recomendados pela Organização Mundial da Saúde, OMS, e pelo Programa VigiAr do Ministério da Saúde (Tabela 1) (Brasil, 2007; WHO, 2006), conforme a linha limite apresentada no gráfico da Figura 8, com a concentração de aproximadamente $50 \mu \mathrm{g} \mathrm{m}^{-3}$. Com relação ao Índice de Qualidade do Ar, IQA, utilizado na cidade do Rio de Janeiro, as concentrações estavam inadequadas, ou má (Tabela 1) (MonitorAR-Rio, 2012).

O MP apresentou sensibilidade para os eventos adversos e para os fatores físicos ambientais escolhidos pois aconteceram altas e baixas concentrações independentes nos dois pontos de amostragem. A formação, a dispersão, a deposição e a intensidade das concentrações desse poluente são dependentes de efeitos complexos localizados.

Como os valores das concentrações desse poluente variam em baixas resoluções, e, ao mesmo tempo estão acima dos índices recomendados, os gestores ambientais desses locais poderão incluir estudos epidemiológicos para pequenos grupos de pessoas em exposição diária durante a jornada de trabalho, por um determinado período de médio prazo, avaliar as adequações na infraestrutura construída que necessita da circulação do ar externo imediatamente próximo para provê ventilação natural de um ambiente interno, exigirem um rigor maior nos contratos de climatizações artificial e natural, incluir parâmetros bioclimáticas nas construções de novos pavilhões, revisar procedimentos de isolamento dos pacientes imunocomprometidos, reavaliar o posicionamento de câmeras de fluxo laminar e os condutos dos filtros HEPA, enfim, atuar nas questões relativas às interações entre os seus ambientes externo e interno considerando que há variação significativas na qualidade do ar.

Sem pretender esgotar o tema aqui tratado, a aplicação de uma análise estatística rigorosa para um número maior de amostras se faz necessário, mesmo com o sacrifício do manejo dos pesados amostradores e de seu alto ruído, que incomodam as pessoas próximas, principalmente, se forem pacientes em pátios de hospitais.

A conclusão que pode ser obtida da observação direta da análise dos resultados é a constatação de que a baixa resolução permitiu verificar diferenças nos valores das concentrações medidas simultaneamente nos dois pontos de amostragem, e o surgimento de eventos adversos amplificadores que são inesperados. Há certa eficiência na resolução da grade horizontal escolhida, pois os resultados mostraram padrões de similaridades e dissimilaridades entre os pares medidos.

Este trabalho pode contribuir para o desafio de um diálogo entre Engenharias Civil e Ambiental, com ênfase em Saúde Pública, no caso de medir MP nas proximidades de um centro hospitalar. Para reforçar esse diálogo seria importante investigar a presença de bioaerossóis patógenos no MP, já que esse poluente é carreador de diversas partículas orgânicas biogênicas, como fungos, pólens, bactérias e vírus, visto que podem acontecer picos de concentrações próximos de janelas, portas, varandas, e, consequentemente, a falta de uma infraestrutura construída preventiva pode levar riscos aos pacientes que já estão imunodeprimidos. Outra contribuição é no campo da Epidemiologia, já que os resultados mostram a necessidade de se desenvolver um estudo de análise de risco para efeitos subcrônico tóxicosistêmico (não carcinogênico) - alergias, crises de asmas - e o slope fator, que é avaliar a doseresposta da ação de uma substância tóxica em miligrama por quilograma de peso corporal dia, em pequenas coortes que frequentam regularmente os reduzidos espaços geográficos exemplificados neste trabalho imersos em corredores de poluentes do ar com a presença de material particulado.

\section{AGRADECIMENTOS}

Para realizar este estudo algumas responsabilidades gerenciais com as questões de segurança, disponibilidade, manejo de insumos e 
controle dos equipamentos foram assumidas. Além disso, realizaram-se parcerias entre o Departamento de Engenharia Ambiental da UERJ e o Departamento de Administração do Instituo Nacional de Infectologia, Ini/Fiocruz/RJ, o Laboratório de Físico-Química do Instituto de Química da Universidade Federal do Rio de Janeiro, IQ/UFRJ, e com o Centro de Estudos da Saúde do Trabalhador e Ecologia Humana, CESTEH, da Escola Nacional de Saúde Pública Sérgio Arouca, Ensp/Fiocruz/RJ, que permitiram a orientação, a seleção do método de pesquisa, as atividades de monitoramento da campanha, guarda dos equipamentos, calibração, instalação, acompanhamento, utilização dos insumos, participação das atividades laboratoriais, antes e depois das campanhas de medição, além da colaboração das pessoas que auxiliaram a campanha.

\section{REFERÊNCIAS BIBLIOGRÁFICAS}

ARTAXO, P. E., GATTI, Luciana V., LEAL, A. M. C., LONGO, K. M., FREITAS, S. R. de, LARA, L., et al. Química atmosférica na Amazônia: A floresta e as emissões de queimadas controlando a composição da atmosfera amazônica. Acta Amazônica, v. 35, n. 2, p. 185 - 196, 2005.

AGOPYAN, V., JOHN, V. M. O desafio de sustentabilidade na construção civil. São Paulo, Blucher, 2011.

ASSOCIAÇÃO BRASILEIRA DE NORMAS TÉCNICAS. NBR 13412: Material particular em suspensão na atmosfera: determinação de particulas inaláveis pelo método de amostrador de grande volume acoplado com um separador inercial de particulas. Rio de Janeiro, 1995.

NBR 9547: Material particulado em suspensão no ar ambiente - determinação da concentração total pelo método de grande volume. Rio de Janeiro, 1997.

BAIRD, C. Química Ambiental. 4a ed.. Porto Alegre, Bookman, 2011.

BATTERMAN, S., CHAMBLISS, S., ISAKOV, V. Spatial resolution requirements for traffic-related air pollutant exposure evaluations. Atmospheric Environment, n. 94, p. $518-528,2014$.
BORGES, A. S. Impacto do refinamento da grade horizontal do modelo fotoquímico $\mathrm{CIT}$ nas estimativas de concentrações de poluentes atmosféricos [Dissertação de Mestrado], Instituto de Astronomia, Geofísica e Ciências Atmosféricas, USP, 2003.

BRAGA, B., HESPANHOL, I., CONEJO, J. G. L., MIERZWA, J. C., BARROS, M. T., SPENCER, M., et al. Introdução à engenharia ambiental: o desavio do desenvolvimento sustentável. 2a ed. São Paulo, Pearson Prentice Hall, 2005.

BRASIL. VIGIAR. Vigilância em Saúde Ambiental Relacionada à Qualidade do Ar Ministério da Saúde VIGIAR. Secretaria de Vigilância em Saúde. Relatório Final - Reunião de Avaliação das Metas 2006 e Pactuação de Ações para 2007. Brasília, 2007.

CASTANHO, A. D. A. A determinação quantitativa de fontes de material particulado na atmosfera da cidade de São Paulo. [Dissertação de Mestrado], Instituto de Física da Universidade de São Paulo, São Paulo, 1999.

CHENG, M. Air pollution causes cancer, World Health Organization agency declares. CTV News Channel. Canadá, 2013. Disponível em <http://www.ctvnews.ca/health/health-headlines/airpollution-causes-cancer-world-health-organizationagency-declares-1.1500668>. Acessado em 15 nov 2014

CHITHRA, V. S. e SHIVA NAGENDRA, S. M. Impact of outdoor meteorology on indoor $\mathrm{PM}_{10}, \mathrm{PM}_{2.5}$ and $\mathrm{PM}_{1}$ concentrations in a naturally ventilated classroom. Urban Climat, 13 nov 2014.

COLTRI, P. P. Ilhas de Calor da estacão de inverno da área urbana do município de Piracicaba, SP. Anais XIII Simpósio Brasileiro de Sensoriamento Remoto, Florianópolis, Brasil, 21-26 abril 2007, INPE, p. 51515157, 2007.

CONSELHO NACIONAL DE MEIO AMBIENTE. CONAMA № 003. Determinação de padrões de qualidade do ar para poluentes atmosféricos passíveis de monitoramento e controle no País; 1990.

ERENO, D. Para evitar flagelos. Revista Pesquisa FAPESP. Ed. 171, p. 16-21, set 2010.

FREITAS, S. P., LONGO, Karla M. SILVA DIAS, M. F. A., SILVA DIAS, P. L.. Emissões de queimadas em ecossistemas da América do Sul. Estudos Avançados, n. 19, v. 53, 2005.

FRONDIZI, C. A. Monitoramento da qualidade do ar: Teoria e Prática. Rio de Janeiro, E-papers, 2008. 
GARTLAND, L. Ilhas de calor : como mitigar zonas de calor em áreas urbanas. Tradução Sílvia Helena Gonçalves. São Paulo, Oficina de Textos, 2010.

GJORV, O. E. Projeto de durabilidade de estruturas de concreto em ambientes de severa agressividade. São Paulo, Oficina de Textos, 2015.

HOEK, G., BEELEN, R., HOOGB, K. de, VENNEAU, D., GULLIVER, J., FISCHER, P., BRIGGS, D. A review of landuse regression models to assess spatial variation of outdoor air pollution. Atmospheric Environment, n. 42, p. 7561-7578, 2008.

ISNARD, A. A. Investigação computacional do escoamento e da dispersão de poluentes atmosféricos sobre topografias complexas. [Tese de Doutorado]. Programa de Pós-Graduação em Engenharia Mecânica. Pontifícia Universidade Católica do Rio de Janeiro, Rio de Janeiro, 2004.

LENZI, E. E FAVEIRO, L. O. B. Introdução à química da atmosfera: ciência, vida e sobrevivência. Rio de Janeiro, LTC, 2009.

LOUREIRO, L. N. Panorama sobre emissões atmosféricas. Estudo de caso: avaliação do inventário emissões atmosféricas para fontes móveis. [Dissertação de Mestrado], Rio de Janeiro, Universidade Federal do Rio de Janeiro, 2005.

MADSEN, C. Modeling the intraurban variability of outdoor traffic pollution in Oslo, Norway-A GA2LEN project. Atmospheric Environment, n. 41, p. 7500-7511, 2007.

MANAHAN, S. E. Fundamentals of Environmental Chemistry. 2a ed. Boca Raton, CRC Press LLC; 2001.

MARTINS, E. M. Estudo teórico-experimental de poluentes atmosféricos primários e secundários no bairro da Tijuca, Praça Saens Peña, Rio de Janeiro. Tese de Doutorado em Físico Química, UFRJ, IQ, 2006..

MENSINK, C. RIDDER, K. De, DEUTSCH, F., LEFEBRE, F., VAN DE VEL, K. Examples of scale interactions in local, urban, and regional air quality modelling. Atmospheric Research, n. 89, p. 351-357, 2008.

MONITORAR-RIO. Secretaria Municipal de Meio Ambiente do Rio de Janeiro. Qualidade do Ar na Cidade do Rio de Janeiro. Relatório da Rede Monitorar-Rio, 2011 - 2012, Rio de Janeiro, 2012.

MORAES, N. O. Anuário do Instituto de Geociências UFRJ. v. 28, n. 2, p. 116-138, 2005.

OPAS - Organização Pan-Americana da Saúde. Organização Mundial da Saúde. Mudanças climáticas e ambientais e seus efeitos na saúde: cenários e incertezas para o Brasil. Ministério da Saúde e Fundação Oswaldo Cruz; 2008. Série Saúde Ambiental 1.
PARAJARA, F. Poluição mata mais que Aids e trânsito juntos em São Paulo. O Globo, São Paulo, 14 de outubro de 2008.

PATTINSON, W. Using mobile monitoring to visualise diurnal variation of traffic pollutants across two nearhighway neighbourhoods. Atmospheric Environment, $\mathrm{n}$. 94, p. $782-792,2014$.

PESTANO, A. F. As faces da nuvem de poeira: Natureza em Cuba. Revista Com Ciência Ambiental, São Paulo, ano 1, n. 4, p. 34-35, outubro 2006.

PIRES, D. O. Inventário de emissões atmosféricas de fontes estacionárias e sua contribuição para poluição do ar na Região Metropolitana do Rio de Janeiro. Dissertação de Mestrado, COPPE, UFRJ, 2005.

PROSPERO, J. M., BLADES, E., MATHISON, G., NAIDU, R. Interhemispheric transport of viable fungi and bacteria from Africa to the Caribbean with soil dust Aerobiologia, v. 20, n. 4, 2004.

RAMOS, R. Diesel veicular provoca 3 mil mortes por ano em São Paulo. Gazeta Mercantil, São Paulo, 5 set. 2008.

RICKLEFS, R. E. Economia da natureza. 5a ed. Guanabara Koogan, Rio de Janeiro, 2003.

SALDIVA, P. Meio ambiente e saúde: o desafio das metrópolis. São Paulo, Ex-Libris Comunicação Integrada, 2010.

SEINFELD, J. H., PANDIS, S. N. Atmospheric chemistry and physics: from air pollution to climate change. $2^{\text {a }} \mathrm{ed}$. New Jersey, EUA, Wiley-Interscience, 2006.

SMARGIASSI, A., BALDWIN, M., PILGER, C., DUGANDZIC, R., BRAUER, M. Small-scale spatial variability of particle concentrations and traffic Levels in Montreal: a pilot study. Science of the Total Environment, n. 338, p, 243251, 2005.

SOLAZZO, E., CAI, X., VARDOULAKIS, S. Improved parameterisation for the numerical modelling of air pollution within an urban street canyon. Environmental Modelling \& Software, n. 24, p. 381-388, 2009.

TASEIKO, O. V., MIKHAILUTA, S. V., PITT, A., LEZHENIN, A. A., ZAKHAROV, Y. V. Air pollution dispersion within urban street canyons. Atmospheric Environment, n. 43, p. 245252, 2009.

THEURER, W. Typical building arrangements for urban air pollution modelling. Atmospheric Environment, $\mathrm{n}$. 33, p. 4057-4066, 1999.

TRIGUEIRO, A. Mundo sustentável: abrindo espaço na mídia para um planeta em transformação. São Paulo, Globo, 2007.

VESILIND, P. A., MORGAN, S. M. Introdução à engenharia ambiental. Tradução da 2a ed. Norte-Americana. São Paulo, Cengage Learning, 2011. 
VORMITTAG, E., RODRIGUES, C. G., COSTA, R. R. da, MIRANDA, M. J. de, BRAGA, A. A., NASCIMENTO, N. C., SALDIVA, P. H. N. Avaliação do impacto da poluição atmosférica no Estado do Rio de Janeiro sob a visão da saúde. Instituto Saúde e Sustentabilidade, 2014 A.

VORMITTAG, E., RODRIGUES, C. G., COSTA, R. R. da, MIRANDA, M. J. de, BRAGA, A. A., NASCIMENTO, N. C., SALDIVA, P. H. N. Monitoramento da qualidade do ar no Brasil. Instituto Saúde e Sustentabilidade, 2014 B.

WHEELER, A. J., SMITH-DOIRON, M., XU, X., GILBERT, N. L., BROOK, j. Intra-urban variability of air pollution in Windsor, Ontario- Measurement and modeling for human exposure assessment. Environmental Research, n. 106, p. 7-16, 2008.

WHO. World Health Organization. Air pollution and cancer. Editors, K. Straif, A. Cohen, J. Samet. IARC Scientific Publications, 161, 2013.

WHO. World Health Organization. WHO Air quality guidelines for particulate matter, ozone, nitrogen dioxide and sulfur dioxide. Summary of risk assessment Global update 2005, Geneva, Switzerland, 2006.

WILSON, J. G. e ZAWAR-REZA, P. Intraurban-scale dispersion modelling of particulate matter concentrations: Applications for exposure estimates in cohort studies. Atmospheric Environment, n. 40, p. 1053-1063, 2005.

WILSON, J. G., KINGHAM, S., PEARCE, J., STURMAN, A. A review of intraurban variations in particulate air pollution: Implications for epidemiological research. Atmospheric Environment, n. 39, p. 6444-6462, 2005. 